EPJ Web of Conferences 116, 04002 (2016)

DOI: $10.1051 /$ epjconf/201611604002

(C) Owned by the authors, published by EDP Sciences, 2016

\title{
Indirect detection of Dark Matter with the ANTARES Neutrino Telescope
}

\author{
Miguel Ardid ${ }^{\mathrm{a}}$ on behalf of ANTARES Collaboration
}

Institut d'Investigació per a la Gestió Integrada de les Zones Costaneres (IGIC), Universitat Politècnica de València, C/ Paranimf 1, 46730 Gandia, Spain

\begin{abstract}
One of the main objectives of the ANTARES neutrino telescope is the search for neutrinos produced in self-annihilation of Dark Matter (DM) particles. The analysis for different sources of DM (Sun, Galactic Center, Earth, ....) or DM models (SUSY, Secluded) will be described and the results presented. The specific advantages of neutrino telescopes in general and of ANTARES in particular will be explained. As an example, the indirect search for DM towards the Sun performed by neutrino telescopes currently leads to more stringent limits on the spin-dependent WIMP-nucleon cross section with respect to existing direct detection experiments.
\end{abstract}

\section{Introduction}

The concept of Dark Matter (DM) has been introduced in order to explain the observations in cosmology and astrophysics which seem to indicate that about $84 \%$ of the matter in the Universe is non-baryonic, non-relativistic and does not interact electromagnetically but only significantly through gravity. One of the most favourite models for DM is the so called Weakly Interacting Massive Particles (WIMPs) that form a halo in which the visible baryonic part of galaxies is embedded. Different candidates for WIMPs attract a great deal of interest, especially those provided by theories based on supersymmetry (SUSY). In some of these models, secondary high-energy neutrinos could be produced from the self-annihilation products, or decays of them. In this paper the indirect searches for DM by detecting high-energy neutrinos coming from the GC, Sun and Earth with the ANTARES neutrino telescope are presented.

\section{Method}

\subsection{The ANTARES neutrino telescope}

The ANTARES neutrino telescope [1] is located in the Mediterranean Sea, at a depth of $2.5 \mathrm{~km}$, about $40 \mathrm{~km}$ off Toulon (France). It consists of 885 Optical Modules (OMs) arranged in a three-dimensional array. The operation principle is based on the detection by these OMs of the Cherenkov light induced by relativistic charged particles, mostly muons produced in interactions of high energy neutrinos in the

\footnotetext{
a e-mail: mardid@fis.upv.es
}

This is an Open Access article distributed under the terms of the Creative Commons Attribution License 4.0, which permits unrestricted use, distribution, and reproduction in any medium, provided the original work is properly cited. 
vicinity of the detector. The OMs are installed along 12 lines anchored to the sea floor and kept vertical by buoys at the top of them. The length of the lines is $450 \mathrm{~m}$ and the distance between the lines is 60-75 $\mathrm{m}$. The position [2] and time [3] information of the photons detected by the OMs can be used to reconstruct the muon, and thus neutrino, direction. The installation of the detector was completed in 2008. In these DM searches data recorded between 2007 and 2012 are used. During this time, the detector consisted of 5 lines for most of 2007 and of successively 8, 9, 10 and 12 lines.

\subsection{Signal and background estimation}

The signal, i.e. the flux of muon neutrinos as a function of their energy arriving at the Earth's surface from the source is computed using Monte Carlo packages and previously obtained results (WimpSim [4] for the Sun and Earth, and [5] for GC). With this, neutrinos for the relevant channels and for DM masses ranging from $25 \mathrm{GeV}$ to $10 \mathrm{TeV}$ are derived. Propagation effects, i.e. oscillations among the three neutrino flavours, neutrino absorption and regeneration, are taken into account by these Monte Carlo packages. There are two sources of background in the ANTARES detector: the muons and neutrinos produced by cosmic rays interacting in the atmosphere. In order to reduce the first one, only upgoing events are selected, since muons cannot traverse the Earth. However, some of these muons (a tiny fraction of the total, but non-negligible number given the flux involved) are misreconstructed as upgoing. This is why further cuts are needed on the quality of the reconstructed track in order to reject them. On the other hand, the atmospheric neutrinos are irreducible background. For the Sun and Galactic Center cases, the background estimations are done by scrambling real data using random times, which reduces the impact of systematic uncertainties. For the Earth, since we look always to vertical tracks, this approach is not possible and background is estimated from simulations.

\subsection{Optimization}

A blind strategy procedure has been followed for the analysis in order to avoid selection biases. This means that the cuts have been selected before looking at the source region. The criterion to choose the cuts has been to optimize (minimize) the average upper limit which can be set for any given DM mass and channel. This has been done using Feldman-Cousins method [6] and using an average effective area that takes into account the livetime of each detector configuration [7].

The optimization is done by scanning the average upper limit for different values of quality reconstruction parameter and search cone angles around the source direction for the binned searches and using a likelihood procedure for unbinned analyses. For each WIMP mass and channel, an optimized set of cuts is found using the blind procedure. Then, real data is looked at and since there were not any significant excess in the searches, limits to DM were derived.

\section{Searches and results}

\subsection{Indirect search for Dark Matter towards the Galactic Centre}

A binned search for high-energy neutrinos coming from the direction of the Galactic Centre has been performed with the event selection criteria to maximise the sensitivity to possible signals produced by the self-annihilation of WIMPs accumulated around the centre of the Milky Way with respect to the atmospheric background for different possible channels: bb, WW, $\tau \tau, \mu \mu, v v$ [8]. Since the number of neutrinos observed is compatible with background expectations, the $90 \%$ C.L. upper limits in terms of the neutrino flux and the velocity averaged annihilation cross-section $\langle\sigma \mathrm{v}\rangle$ are derived for the different WIMP self-annihilation channels. These limits for $\langle\sigma \mathrm{v}\rangle$ are shown to be the most stringent for a neutrino telescope over the WIMP masses $25 \mathrm{GeV}<\mathrm{M}_{\mathrm{WIMP}}<10 \mathrm{TeV}$. As an example, the $90 \%$ 

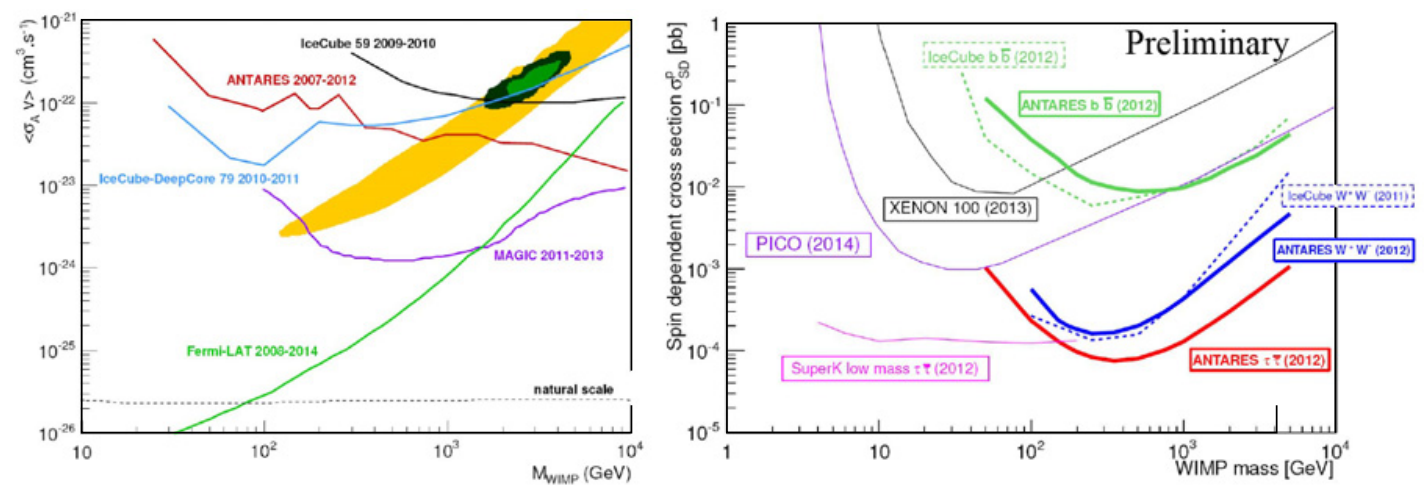

Figure 1. Left: The $90 \%$ C.L. upper limit on the WIMP velocity averaged self-annihilation cross-section, $<\sigma v>$ as a function of the WIMP mass in the range $25 \mathrm{GeV}<\mathrm{M}_{\text {WIMP }}<10 \mathrm{TeV}$ for the WIMP self-annihilation into $\tau^{+} \tau^{-}$, for ANTARES 2007-2012 GC search (red). This is compared to the limits from other experiments. The interpretation of observed electron/positron excesses as DM self-annihilations is also shown as filled areas orange (PAMELA) and green (PAMELA, Fermi-LAT and H.E.S.S). The dashed line indicates the natural scale for which a WIMP is a thermal relic of the early Universe. Figure from [8]. Right: The 90\% C.L. upper limits on the spin-dependent WIMP-proton scattering cross section as a function of the WIMP mass obtained and compared to other results from neutrino telescopes and direct detection experiments. Figure from [9].

C.L. upper limit for $\langle\sigma \mathrm{v}\rangle$ WIMP self-annihilation into $\tau^{+} \tau^{-}$is presented in Fig. 1-left and compared to the limits obtained from other experiments and regions of interest proposed.

\subsection{Indirect search for Dark Matter towards the Sun}

An indirect search for DM towards the Sun has been performed using the unbinned method [9]. Since no excess of data has been observed in the direction of the Sun, limits have been calculated in terms of muon neutrino fluxes and spin-dependent WIMP-nucleon scattering cross sections. Since the Sun is composed mainly of hydrogen indirect searches are more sensitive to the spin-dependent scattering cross section and can surpass even direct detection experiments, such as PICO [10], with the ANTARES limits becoming very competitive for high WIMP mass $(\geq 1 \mathrm{TeV})$, see Fig. 1-right. Direct detection experiments, such as LUX [11], are definitively more competitive for the spin-independent cross section (see Fig. 2).

\subsubsection{Search for Secluded Dark Matter}

Secluded DM is a special scenario where DM annihilates into a pair of non-Standard Model mediators, $\phi$, which subsequently decay into SM particles. It was suggested to explain some experimental observations, such as the positron-electron ratio observed by satellite detectors. In case of meta-stable mediators, the decay products could either be detected directly in the Earth or the final spectrum of neutrino fluxes could be modified significantly. In this search $[12,13]$ two different cases are studied: a) detection of di-muons from mediator decays [14], either directly or indirectly through mediators which decay into di-muons that, in turn, decay into neutrinos, and b) the mediator that directly decays into neutrinos [15]. These results $[12,13]$-the first experimental limits established directly in neutrino telescopes- are more restrictive than those derived in direct detection searches for the case of spindependent interaction for a wide range of lifetimes of the meta-stable mediator. 


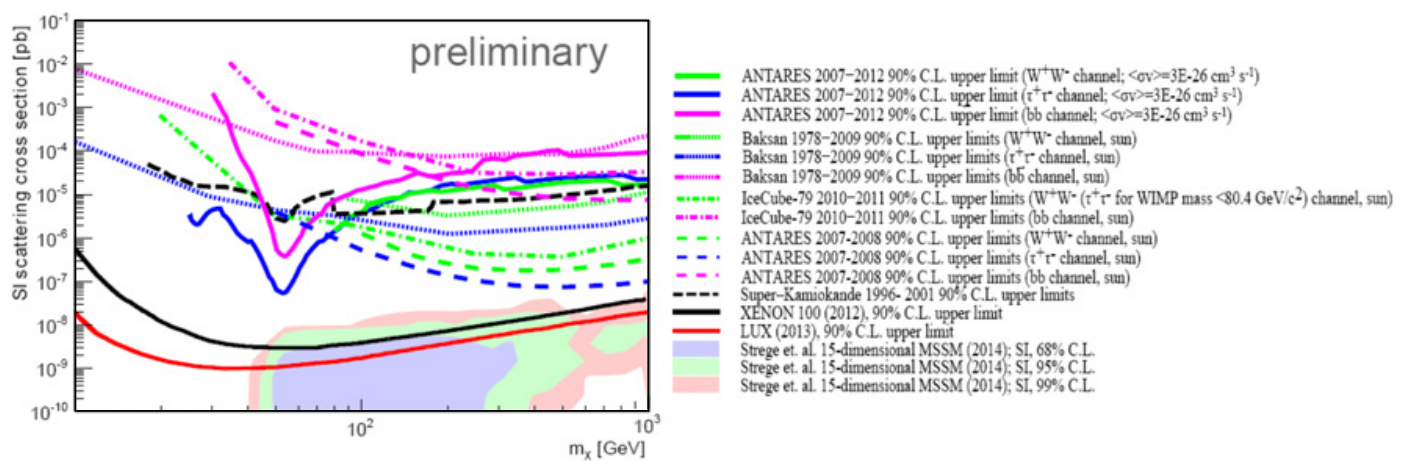

Figure 2. 90\% CL upper limits on $\sigma \mathrm{SI}$ as a function of the WIMP mass for $<\sigma \mathrm{v}>=3 \cdot 10^{-26} \mathrm{~cm}^{3} \mathrm{~s}^{-1}$ and WIMP pair annihilation to $100 \%$ into either $\tau^{+} \tau^{-}, \mathrm{W}^{+} \mathrm{W}^{-}$or bb, for ANTARES (Earth) 2007 - 2012, compared to other results from neutrino telescope, direct detection experiments and MSMM predictions. Figure from [16].

\subsection{Indirect search for Dark Matter towards the Earth Centre}

WIMPs could also scatter with Earth matter and be trapped. An indirect search for DM can be performed with ANTARES by looking for an excess of the neutrino flux from the Earth's centre [16]. The spectrum of the neutrino flux from the Earth would depend on the WIMP mass, the annihilation channel, the spin independent scattering cross section and the thermally averaged annihilation cross section of the WIMPs. No significant excess over the background expectation has been found and 90\% CL upper limits on the WIMP self-annihilation rate were set as a function of the WIMP mass for different channels. These were translated to limits on the spin independent scattering cross section. Figure 2 shows the results of this search and compare them to other searches with neutrino telescopes and direct detection experiments. Although direct detection experiments put more stringent constrains, there might be some differences between experiments using different targets and technologies. In this sense, this kind of search can be complementary to direct detection.

We acknowledge the financial support of the Spanish Ministerio de Economía y Competitividad, Grants FPA2012-37528-C02-02, and Consolider MultiDark CSD2009-00064, of the Generalitat Valenciana, Grants ACOMP/2015/175 PrometeoII/2014/079 and of the European FEDER funds.

\section{References}

[1] M. Ageron, et al., ANTARES Collaboration, Nucl. Instrum. and Meth. A 656, 11 (2011)

[2] M. Ardid, for the ANTARES Collaboration, Nucl. Instrum. and Meth. A 602, 174 (2009). S. Adrián-Martínez et al., ANTARES Collaboration, J. Instrum. 7, T08002 (2012)

[3] J.A. Aguilar et al., ANTARES Collaboration, Astropart. Phys. 34, 539 (2011)

[4] Mattias Blennow, Joakim Edsjo, Tommy Ohlsson, JCAP 01, 021 (2008). J. Edsjo, WimpSim Neutrino Monte Carlo, http://www.fysik.su.se/ edsjo/wimpsim/

[5] M. Cirelli, et al., JCAP 03, 051 (2011)

[6] G.J. Feldman, R.D. Cousins, Phys. Rev. D 57, 3873(1998)

[7] S. Adrián-Martínez et al., ANTARES Collaboration, JCAP 11, 032 (2013)

[8] S. Adrián-Martínez et al., ANTARES Collaboration, JCAP 10, 068 (2015)

[9] C. Tönnis, for the ANTARES collaboration, PoS (ICRC2015) 1207

[10] C. Amole et al., PICO Collaboration, Phys. Rev. Lett. 114, 231302 (2015)

[11] D.S. Akerib et al., LUX Collaboration, Phys. Rev. Lett., 112, 091303 (2014) 
[12] M. Ardid and C. Tönnis, for the ANTARES collaboration, PoS (ICRC2015) 1212

[13] S. Adrián-Martínez. Ph. D. Thesis. Universitat Politècnica de València (2015)

[14] P. Meade, S. Nussinov, M. Papucci, T. Volansky. J. High Energy Phys. 06, 29 (2010)

[15] N.F. Bell, K. Petraki. JCAP 04, 003 (2011)

[16] A. Gleixner and C. Tönnis, for the ANTARES collaboration, PoS (ICRC2015) 1110 